\title{
Las diásporas de los árboles y arbustos nativos de México: posibilidades y limitaciones de uso en programas de reforestación y desarrollo agroforestal
}

\author{
Aníbal Niembro Rocas
}

\section{RESUMEN}

Se analiza la importancia de las diásporas de los árboles y arbustos nativos de México como medios de propagación de árboles y arbustos con fines de reforestación y desarrollo agroforestal, resaltando las causas que han limitado la utilización de esta forma de germoplasma en los viveros forestales. Se concluye la necesidad de fortalecer las investigaciones en materia de fenología, estructura y morfología de diásporas, latencia y viabilidad, germinación y desarrollo inicial de plántulas para aquellas especies potencialmente importantes de las nueve mil existentes en el territorio nacional.

PALABRAS CLAVE:

Árboles, arbustos, diásporas, reforestación, desarrollo agroforestal.

\begin{abstract}
The importance of the diaspores of the trees and native shrubs of Mexico like materials of propagation of plants with reforestation ends and agroforestry development is analyzed, highlighting the causes that have limited the use this form of germplasm in forest nurseries. It is concluded the how necessary is to strengthen research on phenology, structure and diaspore morphology, dormancy and viability, germination and initial development of seedlings for those species potentially important of the nine thousand growing in the national territory.
\end{abstract}

KEY WORDS:

Trees, shrubs, diaspores, reforestation, agroforestry development. 


\section{INTRODUCCIÓN}

En los últimos 50 años la cubierta vegetal de México se ha reducido significativamente, en particular durante los años 70's cuando el Programa Nacional de Desmontes (PRONADE) dictaminó la incorporación de 25 millones de hectáreas de aptitud forestal a actividades agropecuarias. Las comunidades vegetales mayormente impactadas por los desmontes fueron los bosques tropicales y en menor cuantía los bosques de zonas montañosas y los matorrales xerófilos. Aunado a lo anterior, los incendios, las cortas clandestinas y la sobreexplotación de especies valiosas han contribuido a la disminución de la cubierta forestal del país. De acuerdo con el diagnóstico realizado dentro del Plan Estratégico Forestal México 2025 (SEMARNAT, 2001) la superficie total arbolada del país en 1994 era de 56.8 millones de hectáreas. Para el año 2000 dicha superficie se había reducido a 53 millones de hectáreas a un promedio de deforestación de unas 600 mil hectáreas anuales. La pérdida de la cubierta vegetal ha traído como consecuencia la falta de agua en varias regiones del país; el deslizamiento de laderas de cerros e inundaciones durante la temporada de lluvias afectando a numerosas comunidades serranas; la sedimentación y desbordamiento del cauce de los ríos y la disminución de las posibilidades agropecuarias debido a la pérdida paulatina de tierra fértil.

Además de cambiar dramáticamente el uso del suelo, la deforestación ha ocasionado profundas alteraciones en el paisaje mexicano. Hoy en día los cambios más notables que se observan en diversas regiones del país son la presencia de islas de vegetación primaria y secundaria rodeadas de terrenos degradados por la erosión del viento y la lluvia, cultivos agrícolas diversos, potreros, asentamientos humanos irregulares, plantaciones forestales generalmente coetáneas y monoespecíficas, muchas de ellas establecidas con especies exóticas, desarrollos turísticos y parques industriales principalmente.

La conversión gradual de los bosques mexicanos en islas de vegetación ha dado como resultado la destrucción de algunos hábitats y la contaminación de otros. Los corredores biológicos, que en otras épocas formaban un paso continuo para la fauna silvestre en las serranías y planicies costeras del Golfo y del Pacífico, hoy en día se encuentran fragmentados. Numerosas especies de animales y plantas permanecen aisladas y con ello sus posibilidades de cruzamiento, intercambio genético y evolución se han visto disminuidas al haberse reducido su variación natural y nichos ecológicos.

Con el propósito de ayudar a revertir el deterioro causado por los desmontes, incendios forestales, tala clandestina, pastoreo, plagas y enfermedades el Gobierno Federal creó en 1995 el Programa Nacional de Reforestación (PRONARE), el cual fue concebido como una herramienta para recuperar e incrementar la cubierta vegetal del país, así como restaurar y proteger los ecosistemas dañados antes de que los recursos remanentes de vegetación natural se agoten por completo. A partir de esa fecha las plantaciones forestales con fines de protección y restauración, conservación y arborización urbana, entre otras, se han incrementado en el territorio nacional.

A través del PRONARE, desde su inicio hasta el año 2000 , se han producido en el país más de 1,500 millones de árboles para reforestar una superficie de 571 mil hectáreas (SEMARNAT, 2001). Como consecuencia de lo anterior, las necesidades de diásporas de variadas especies de árboles y arbustos tanto nativos como exóticos también han aumentado considerablemente. Desafortunadamente, de las 484 especies de plantas utilizadas por el PRONARE a nivel nacional, sólo el $31.4 \%$ corresponde a 
árboles y arbustos nativos $(9.7 \%$ son coníferas y $21.7 \%$ son latifoliadas de clima templado y tropical), el resto (68.6\%) está representado por palmas y cícadas y por especies exóticas las cuales resultan extrañas a los hábitats que se están reforestando (SEMARNAP, 1999).

Las razones por las cuales no se utiliza un mayor número de árboles y arbustos de las aproximadamente 9000 especies existentes dentro de la flora nacional son diversos. Algunas de las causas que han limitado la propagación y el uso de muchos de los árboles y arbustos nativos en programas de reforestación, restauración ecológica y desarrollo agroforestal obedecen al desconocimiento de la biología y manejo de las diásporas que producen.

\section{Las diásporas de los árboles y arbustos en la práctica de la silvicultura y agroforestería}

Las plantas superiores han desarrollado, en el curso de su evolución, diversos órganos autónomos producidos por vía agámica y sexual. Dichos órganos al separarse de la planta madre y ser diseminados por el hombre, el viento, el agua 0 los animales silvestres y domésticos, tienen la capacidad de originar nuevos individuos similares a la planta que los engendró. Cuando estos órganos son de origen agámico o asexual reciben el nombre de "propágulos" (del latín propagulum, propagar o multiplicar vegetativamente). Cuando son de origen sexual se conocen con los nombres de "diásporas" (del griego $\delta 1 \alpha \sigma \pi \mathrm{o} \alpha$, diseminación, dispersión) o "disemínulos" (del latín disseminare, sembrar) y están formados por uno o varios embriones y un complejo orgánico acompañante.

De acuerdo con van der Pijl (1969), las diásporas o disemínulos de los árboles y arbustos están representados por: 1) embriones desnudos; 2) semillas producidas en frutos carnosos como bayas, pomos y hesperidios, así como en frutos secos dehiscentes como cápsulas, legumbres, folículos, silicuas, etc. 3) pequeños frutos indehiscentes como nuececillas, aquenios, esquizocarpos y sámaras y, 4) partes de frutos drupáceos como pirenos los cuales conjuntamente con su contenido de semillas constituyen por sí mismos una sola unidad de dispersión. En la práctica, los tipos de diásporas mencionados se conocen común y colectivamente con el nombre de "semillas" independientemente de su origen y estructura (Fig.1).

Las diásporas de los árboles y arbustos son reservorios de material genético y constituyen una de las formas más importantes de germoplasma vegetal (King, 1972). La regeneración periódica y de manera natural de numerosas especies de plantas leñosas de importancia actual y potencial se realiza a través de estos órganos. Gracias a su capacidad para originar una nueva planta, las diásporas funcionan como un puente de unión entre una generación y la siguiente. Para los habitantes de comunidades rurales localizadas en regiones cuyos recursos de vegetación se han reducido o agotado por los desmontes y quienes tienen la necesidad de recorrer diariamente grandes distancias en busca de leña, forraje, materiales de construcción, alimentos y sustancias medicinales, las diásporas de los árboles y arbustos de uso múltiple que crecen localmente significan una esperanza de vida en sus lugares de origen. La propagación sexual de plantas por medio de diásporas permite el desarrollo de programas de reforestación, restauración ecológica y aplicación de variadas tecnologías en sistemas agroforestales y silvopastoriles. Dicho en otras palabras, las diásporas de numerosas especies de árboles y arbustos son necesarias para satisfacer las necesidades de alimentación, vestido, salud, vivienda, energía y esparcimiento de millones de seres humanos cuyos requerimientos por productos y servicios de origen vegetal se incrementa diariamente. 


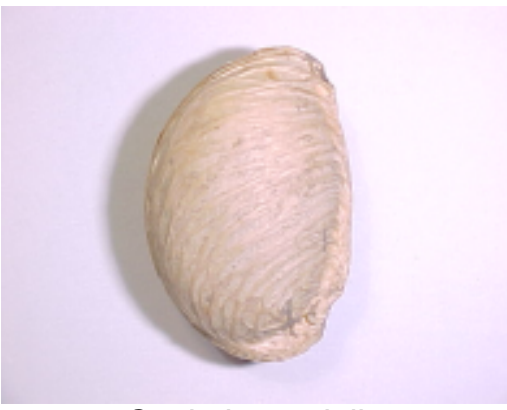

Casimiroa edulis

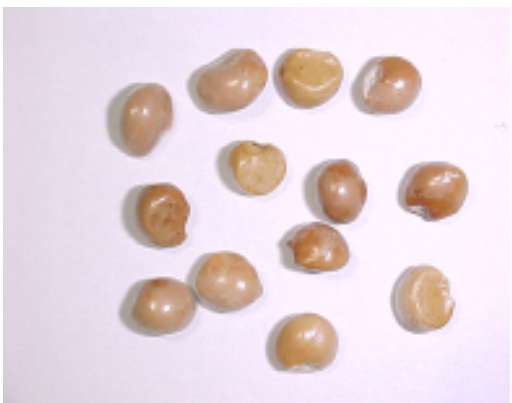

Turpinia insignis

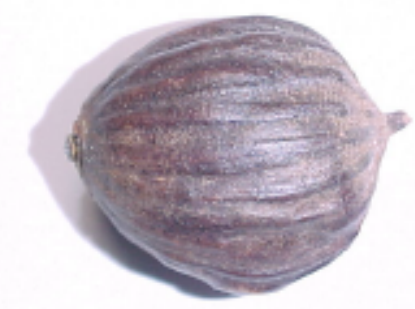

Alfaroa mexicana

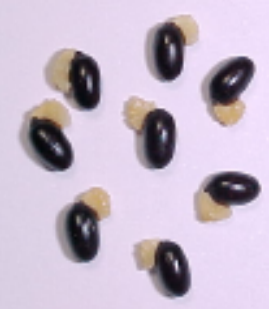

Chirantodendron pentadactylon

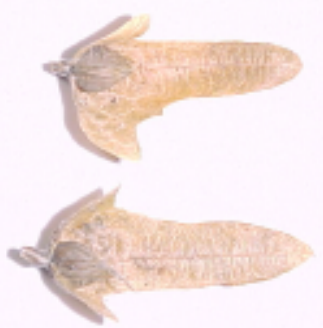

Carpinus caroliniana

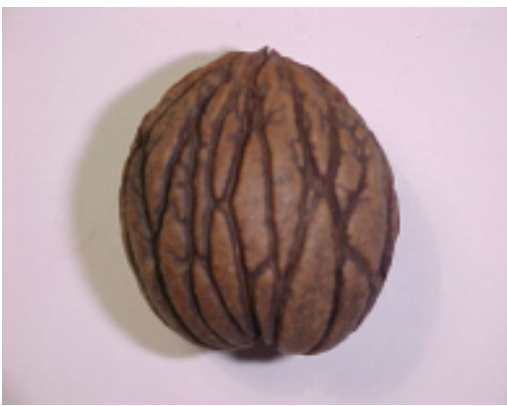

Juglans olanchana

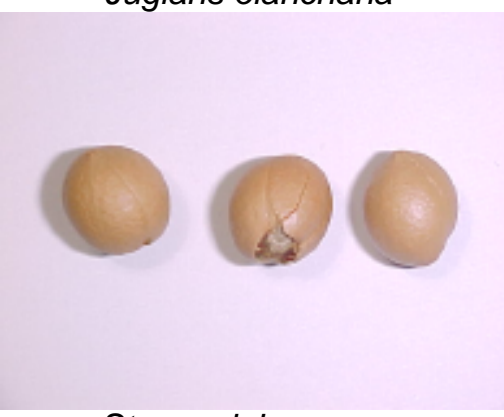

Styrax glabrescens

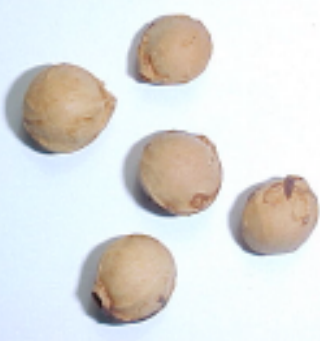

Prunus serotina

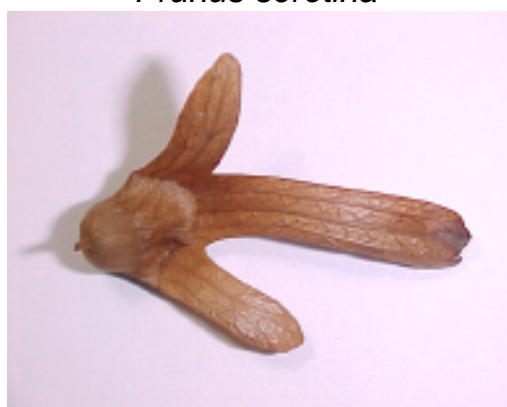

Oreomunnea mexicana
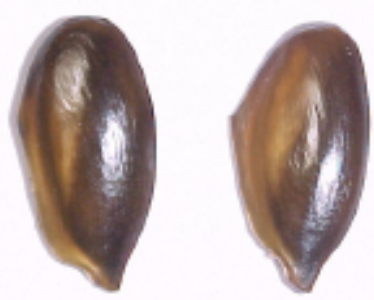

Manilkara zapota

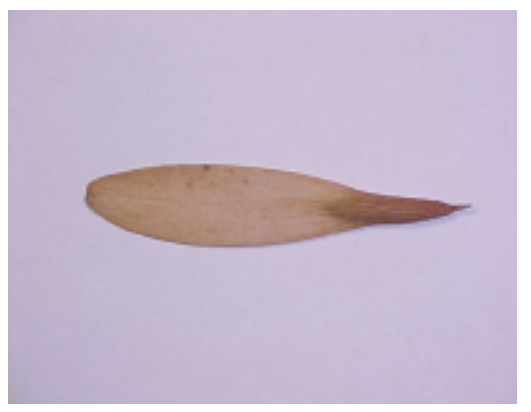

Fraxinus uhdei

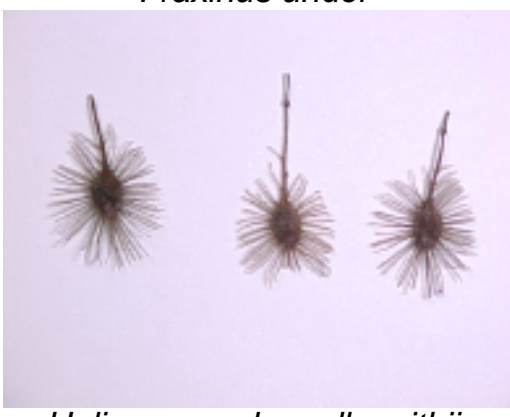

Heliocarpus donnell-smithii

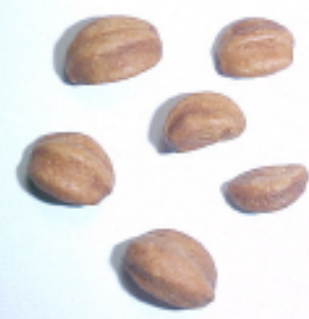

Crataegus pubescens

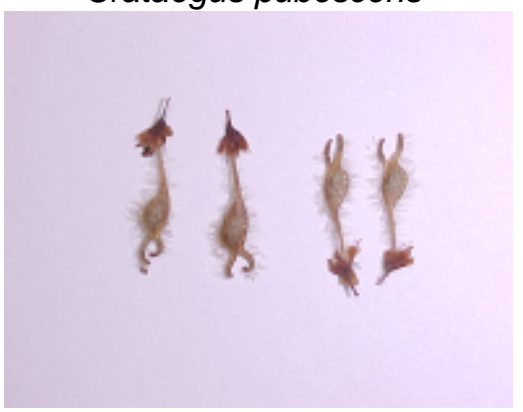

Ulmus mexicana

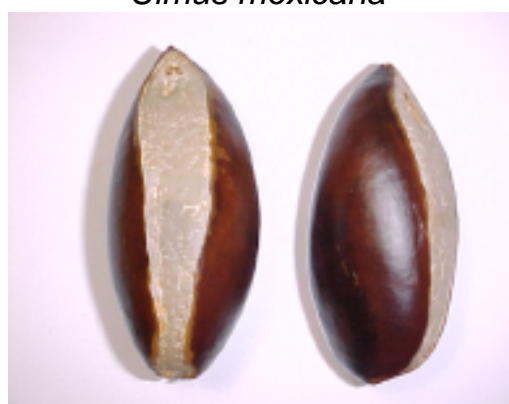

Pouteria sapota

Figura 1. Diásporas de algunos árboles nativos de México comunes en regiones cálidohúmedas y templadas de valor actual y potencial en reforestación y desarrollo agroforestal 
Para silvicultores, horticultores, restauradores del medio ambiente, dasónomos urbanos y viveristas forestales, las diásporas de los árboles y arbustos representan un poderoso instrumento para contrarrestar los efectos deletéreos ocasionados por la destrucción de la cubierta vegetal en todas sus formas y también para elevar la productividad y el valor de la tierra por unidad de superficie.

Con el aumento de los programas de reforestación, forestación, restauración ecológica, desarrollo agroforestal y silvopastoril en diversas regiones del planeta, la demanda por diásporas de plantas leñosas, en particular de árboles y arbustos de uso múltiple se ha incrementado notablemente, sobre todo en aquellos países en donde las plantaciones forestales son una prioridad nacional (Burley y von Carlowitz, 1984; Burley, 1985; von Carlowitz, 1991).

La propagación por medio de diásporas constituye hoy en día la manera más rápida, fácil y barata de obtener materiales de plantación en grandes cantidades destinados a recuperar, ampliar y conservar la cubierta vegetal como una medida para contrarrestar los daños ambientales ocasionados por la deforestación y contribuir al desarrollo social sustentable en aquellas regiones en donde los bosques naturales han desaparecido 0 se encuentran fragmentados cuando se compara con otras formas de propagación como estacas, acodos, injertos y cultivo de tejidos.

\section{Importancia de los árboles nativos de México en la reforestación, restauración ecológica y desarrollo agrosilvopastoril}

La pronunciada orografía del territorio nacional, la presencia de una amplia variedad de climas y suelos y su posición geográfica en el Continente Americano como zona de transición entre las regiones neártica y neotropical, han hecho de México un país con numerosas facetas y contrastes que ocupa el tercer lugar entre los países con mayor diversidad biológica en el mundo (Williams et al., 1992). La flora nativa está compuesta de tres elementos principales: el boreal, procedente del Hemisferio Norte; el meridional, originario del Hemisferio Sur, y el autóctono o endémico. Este hecho ha favorecido el desarrollo de una flora fanerogámica rica y variada representada en su conjunto por unas 220 familias, 2,410 géneros y aproximadamente 22,000 especies, las cuales se presentan en mayor o menor medida en los bosques tropicales perennifolios, bosques tropicales subcaducifolios, bosques tropicales caducifolios, bosques espinosos, matorrales xerófilos, pastizales, bosques de encinos, bosques de coníferas y bosques mesófilos de montaña (Rzedowski, 1978, 1992a).

De todas las especies vegetales que conforman los bosques de México, los árboles y los arbustos son las formas biológicas más sobresalientes e importantes. De acuerdo con Rzedowski (1992b) en el territorio nacional vegetan alrededor de 2,500 especies de árboles y unas 6,500 especie de arbustos nativos. Ambos tipos de plantas constituyen en su conjunto un reservorio de recursos bióticos de valor actual y potencial por los múltiples productos y beneficios ambientales que de ellos se derivan, motivo por el cual su presencia y permanencia es fundamental para contrarrestar los efectos de la deforestación y garantizar la sustentabilidad de la tierra (Vázquez et al., 1999).

La importancia de algunas especies de árboles y arbustos nativos como fuente de diversos productos de uso diario y servicios ambientales era bien conocida por las diversas culturas que florecieron en Mesoamérica antes de la conquista. Hoy en día, un ejemplo vivo de la utilización de los árboles y arbustos con diversos fines se encuentra en la Península de Yucatán, en donde los campesinos de origen maya utilizan de manera tradicional la madera de diversas 
especies locales para la construcción de sus viviendas y cultivan en sus huertos familiares más de 92 especies de plantas leñosas de uso múltiple, muchas de las cuales (el $61 \%$ ) proceden directamente de la flora nativa de la región (Barrera, 1980; Villiers et al., 1981; Gómez y Kauz, 1990).

Muchos de los árboles y arbustos nativos de México pueden ser potencialmente valiosos para ser utilizados en programas de reforestación, restauración ecológica y desarrollo agrosilvopastoril, tolerando variadas formas de manejo y cultivo en uno o más sistemas de uso de la tierra en regiones cálidohúmedas, templado-frías, áridas y semiáridas. Desafortunadamente, en la práctica de las actividades mencionadas, únicamente se propaga una fracción muy pequeña del total de la biodiversidad de los árboles y arbustos nativos de México en los viveros forestales.

\section{Posibilidades de utilización}

De manera tradicional las diásporas de un número muy reducido de árboles y arbustos nativos de México se han venido utilizando con fines de reforestación, restauración ecológica y desarrollo agrosilvopastoril. Las causas que han limitado la propagación y el uso de un mayor número de especies obedecen, entre otras cosas, al desconocimiento de la biología y manejo de las diásporas que producen, existiendo profundos vacíos de información en algunos aspectos como los que a continuación se mencionan:

Fenología. El conocimiento de las épocas en las cuales florecen y fructifican los árboles y arbustos de numerosas especies es escaso. La falta de información fenológica ha limitado la colecta en tiempo y forma de las diásporas de variadas especies silvestres potencialmente útiles de diversas regiones del país. Algunos estudios sobre fenología de árboles y arbustos de México han sido publicados por Patiño (1973) y Juárez et al. (1989).
Estructura y morfología. Las diásporas de las especies que se utilizan periódicamente en los viveros forestales son bien conocidas por los técnicos y no tienen problemas para ser identificadas durante su recolección, manejo post-cosecha, siembra, venta e intercambio. Sin embargo, las diásporas de numerosas especies silvestres presentan problemas para su identificación debido a que su estructura y morfología no se encuentra descrita ni ilustrada en manuales especializados que faciliten su reconocimiento de manera aislada. Son pocos los estudios sobre caracterización morfológica de diásporas de árboles y arbustos de México que se han realizados (Niembro, 2000).

Latencia y viabilidad. Las diásporas que producen los árboles que vegetan en las regiones cálido-húmedas, por lo general, presentan la tendencia a germinar rápidamente y permanecer viables por un periodo muy corto. Por el contrario, las diásporas de los árboles que se encuentran en las regiones templadofrías, áridas y semiáridas se caracterizan por presentar latencia y tener una viabilidad larga. Sin embargo, para numerosas especies nativas no se conoce con exactitud si las diásporas que producen presentan algún tipo de latencia ni tampoco la duración de su viabilidad bajo condiciones naturales. Algunos estudios orientados a conocer y manejar la latencia y viabilidad de las diásporas de diversas especies de árboles y arbustos de México se encuentran en Moreno (1976 a y b), Vega et al. (1981), Camacho (1990, 1994, 2000).

Germinación. El conocimiento de los patrones de germinación, así como la velocidad y capacidad germinativa de las diásporas de los árboles y arbustos es fundamental para manejar su regeneración natural y propagación en vivero. Desafortunadamente, este conocimiento dista mucho de estar completo para numerosas especies nativas poco utilizadas en los programas convencionales de reforestación. Algunos estudios llevados a cabo para conocer las 
condiciones y el tiempo requerido para germinar de las diásporas de diversas especies de árboles nativos de México han sido efectuados por López y Vázquez (1976), De la Garza y Ortega (1981), Pedraza (1998), Parraguirre (1988, 1994).

Desarrollo inicial de plántulas. Las plántulas de los árboles y arbustos presentan durante sus etapas iniciales de crecimiento y desarrollo, características morfológicas propias de la especie a la que pertenecen. Dichas características van desapareciendo gradualmente conforme la plántula alcanza posteriores estadios en el curso de su ciclo de vida. En la práctica el conocimiento de las características morfológicas que presentan las plántulas después de su germinación es fundamental para evaluar la regeneración natural de las diversas especies que vegetan en un sitio determinado y controlar la densidad de siembra y espaciamiento entre plantas en los viveros forestales. Este conocimiento es básicamente inexistente para nuestras especies, con la notable excepción del estudio de Del Amo (1979).

\section{CONCLUSIONES}

En términos generales se puede decir, sin temor a equivocarse, que las posibilidades de uso de las diásporas de los árboles y arbustos nativos de México para la producción masiva de plantas con fines de reforestación y desarrollo agroforestal está limitada hoy en día a un grupo muy reducido de especies como el cedro rojo, la caoba, el maculís y algunas especies de pinos, principalmente, las cuales han sido utilizadas cotidianamente en los viveros forestales. Las causas que han limitado la incorporación de árboles y arbustos nativos potencialmente valiosos en los programas de reforestación y desarrollo agroforestal obedecen en cierta medida al desconocimiento parcial o total de la biología y manejo de las diásporas que producen.
En el territorio nacional vegetan alrededor de 2,500 especies de árboles y unas 6,500 especies de arbustos muchos de los cuales por su importancia ecológica y económica tienen que ser gradualmente incorporados a los programas de reforestación, restauración ecológica y desarrollo agrosilvopastoril. Sin embargo, para que su incorporación tenga el éxito esperado se tienen que desarrollar una serie de estudios que permitan conocer con mayores detalles su fenología, la estructura y morfología de las diásporas que producen, así como los tipos de latencia que presentan, la duración natural de su viabilidad, el tipo y requerimientos de germinación y las características morfológicas de sus plántulas durante las primeras etapas de su ciclo de vida.

\section{REFERENCIAS}

Barrera M., A.. 1980. Sobre la unidad de Habitación tradicional campesina y el manejo de recursos bióticos en el área maya yucatanense. Biótica 5(3): 115-129.

Burley, J. 1985. Global needs and problems of the collection, storage and distribution of multipurpose tree germplasm. Science and Practice of Agroforestry 2. International Council for Research in Agroforestry (ICRAF). Nairobi, Kenya. 179 p.

Burley, J. y P.G. von Carlowitz. 1984. Multipurpose tree germplasm. International Council for Research in Agroforestry (ICRAF). Nairobi, Kenya. 298 p.

Camacho M., F. 1990. Eliminación de la dormición de semillas de capulín (Prunus serotina ssp capuli (Cav.) Mc Vaugh). Ciencia Forestal, México. 15(67): 63-74.

Camacho M., F. 1994. Dormición de semillas; causas y tratamientos. Editorial Trillas, México. 125 p. 
Camacho M., F. 2000. Dormición y quiescencia en el manejo de las semillas forestales. Gaceta de la Red Mexicana de Germoplasma Forestal. SEMARNAP. México, D. F. 4: 7-22.

De la Garza L. de L., P. y C. Ortega. 1981. Efecto de la temperatura sobre la germinación de cinco especies tropicales: 281-295. In: Memoria de la Reunión sobre Semillas Forestales Tropicales. Tomo I. Instituto Nacional de Investigaciones Forestales. SARH-SFF. México, D. F.

Del Amo, S. 1979. Clave para plántulas y estados juveniles de especies primarias de una selva alta perennifolia en Veracruz, México. Biótica 4(2): 59-108.

Gómez P., A. y A. Kaus. 1990. Traditional management of tropical forest in México. p: 45-64. In: Alternatives to deforestation: Steps toward sustainable use of the Amazon rain forest. A.B. Anderson (Ed.). Columbia Univ. Press. Nueva York.

Juárez G., V., A. Espinosa B. y O. Cedeño S. 1989. Observaciones fenológicas en 70 especies forestales y su importancia en la producción. p: 874882. In: Memoria del Congreso Forestal Mexicano 1989. Tomo II. Gob. del Estado de México. Toluca, Edo. de México.

King, R.C. 1972. A dictionary of genetics. $2^{\text {nd }}$. ed. Oxford University Press. Londres. 337 p.

Moreno C., P. 1976a. Viabilidad de semillas de árboles tropicales y templados: Una revisión bibliográfica. p: 471-526. In: Regeneración de Selvas. A. Gómez P., C. Vázquez Y. S. del Amo y A. Butanda (Eds.). INIREB. Consejo Nacional Para la Enseñanza de la Biología. CECSA.
Moreno C., P. 1976b. Latencia y viabilidad de semillas de vegetación primaria. p: 527-548. In: Regeneración de Selvas. A. Gómez P., C. Vázquez Y., S. del Amo y A. Butanda (Eds.). INIREB. Consejo Nacional para la Enseñanza de la Biología. CECSA.

López Q., M.M. y C. Vázquez Y. 1976. Estudio sobre germinación de semillas en condiciones naturales controladas. p: 250-262. In: Regeneración de Selvas. A. Gómez P., C. Vázquez Y., S. del Amo y A. Butanda (Eds.). INIREB. Consejo Nacional para la Enseñanza de la Biología. CECSA.

Niembro R., A. 2000. Estructura y morfología de diásporas de árboles y arbustos nativos e introducidos comunes en la República Mexicana. Tesis de Maestría en Ciencias Forestales. División de Ciencias Forestales. Universidad Autónoma Chapingo. Chapingo, Edo. de México. 382 p.

Parraguirre L., C. 1988. Germinación de semillas de especies de vegetación primaria y secundaria (estudio comparativo). Tesis Profesional. Universidad Autónoma Chapingo. Chapingo, Edo. de México.. 89 p.

Parraguirre L., C. 1994. Germinación de las semillas de trece especies forestales comerciales de Quintana Roo. p.: 6780. In: Memoria del Taller Madera, Chicle, Caza y Milpa. Contribuciones al Manejo Integral de las Selvas de Quintana Roo, México. Laura K. Snook y A. Barrera de J. (Eds.). PROAFT, INIFAP, USAID, WWF-US. Chetumal, Quintana Roo, México.

Patiño V., F. 1973. Floración, fructificación y recolección de conos y algunos aspectos sobre semillas de los pinos mexicanos. Bosques y Fauna 10(4): 20-30. 
Pedraza P., R.A. 1998. Germinación de semillas de especies arbóreas colectadas en áreas boscosas de la región de Xalapa, Veracruz. Foresta Veracruzana 1(1): 13-18.

Rzedowski, J. 1978. Vegetación de México. Ed. Limusa. México, D.F. 432 p.

Rzedowski, J. 1992a. Diversidad y orígenes de la flora fanerogámica de México. p: 313-335. In: Diversidad Biológica de Iberoamérica. Acta Zoológica Mexicana. G. Halffter S. (Compilador). Primera Edición. Instituto de Ecología, A. C. Xalapa, Veracruz. México.

Rzedowski, J. 1992b. El endemismo en la flora fanerogámica mexicana: una apreciación analítica preliminar. p:. 337-359. In: Diversidad Biológica de lberoamérica. Acta Zoológica Mexicana. G. Halffter (Compilador). Primera Edición. Instituto de Ecología, A. C. Xalapa, Veracruz. México.

SEMARNAP. 1999. Relación de especies que se utilizan en el PRONARE y su clave de identificación en el SIRECONABIO. Gaceta de la Red Mexicana de Germoplasma Forestal. Secretaría de Medio Ambiente, Recursos Naturales y Pesca 2: 39-49.

SEMARNAT, 2001. Plan estratégico forestal para México 2025. Comisión Nacional Forestal. Secretaría de Medio Ambiente y Recursos Naturales. México, D. F. 142 p. van der Pijl, L. 1969. Principles of dispersal in higher plants. SpringerVerlarg, Nueva York. 153 p.

Vázquez Y., C., A.I. Bátis M., M.I. Alcocer S., M. Gual D. y C. Sánchez D. 1999. Árboles y arbustos nativos potencialmente valiosos para la restauración ecológica y la reforestación. Instituto de Ecología. UNAM. CONABIO. México, D.F. 311 p.

Vega E., C., F. Patiño V. y A.A. Rodríguez y P. 1981. Viabilidad de semillas en 72 especies forestales tropicales almacenadas al medio ambiente. p:. 325-345. In: Memoria de la Reunión sobre Semillas forestales tropicales. Tomo I. Instituto Nacional de Investigaciones Forestales. SARHSFF. México, D. F.

Villiers R., L., R.M. López F. y A. Barrera. 1981. La unidad de habitación tradicional campesina y el manejo de los recursos bióticos en el área maya yucatanense. Biótica 6 (3): 293-323.

von Carlowitz, P. G. 1991. Multipurpose trees and shrubs. Sources of seeds and inoculants. International Council for Research in Agroforestry (ICRAF). Nairobi, Kenya. 328 p.

Williams L., G., G. Halffter S. y E. Ezcurra. 1992. Estado de la biodiversidad en México. p.p. 285-311. In: Diversidad Biológica de Iberoamérica. Acta Zoológica Mexicana. G. Halffter (Compilador). Primera Edición. Instituto de Ecología, A. C. Xalapa, Veracruz. México.»

1 Productos Forestales y Conservación de Bosques. Instituto de Ecología, A. C. Apdo. Postal 63.91000 Xalapa, Veracruz. México. c.e.: anibal@ecologia.edu.mx

Manuscrito recibido el 12 de julio de 2001

Aceptado el 17 de agosto de 2001

Este documento se debe citar como:

Niembro R., A. 2001. Las diásporas de los árboles y arbustos nativos de México: posibilidades y limitaciones de uso en programas de reforestación y desarrollo agroforestal. Madera y Bosques $7(2): 3-11$. 